\title{
Síntesis y sinterización de soluciones sólidas de cromita de lantano obtenidas por el método de coprecipitación en medio alcalino
}

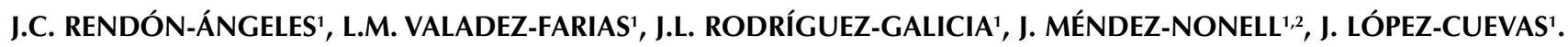 \\ ${ }^{1}$ Centro de Investigación y de Estudios Avanzados del IPN, Unidad Saltillo, Apdo. Postal 663, Saltillo 25000, Coah, México. \\ ${ }^{2}$ Centro de Investigación en Química Aplicada, Saltillo 25100, Coah, Mexico.
}

\begin{abstract}
En el presente trabajo se prepararon tres soluciones sólidas de cromita de lantano del tipo $\mathrm{La}_{0.8} \mathrm{Ca}_{02} \mathrm{Cr}_{1-\mathrm{x}} \mathrm{Al} \mathrm{O}_{3^{\prime}}$ donde $\mathrm{Al}=5$ y $10 \%$ molar, y una de composición $\mathrm{LaCr}_{0.9} \mathrm{Al}_{0.1} \mathrm{O}_{3}$. La preparación del gel precursor se llevó a cabo mezclando soluciones de $\mathrm{Cr}\left(\mathrm{NO}_{3}\right)_{3}$ y $\mathrm{AlCl}_{3}$ en dos diferentes relaciones volumétricas (9.5:0.5 y 9:1). El pH de la solución se ajustó a un valor de 13 mediante la adición del medio de coprecipitación (solución de $\mathrm{NaOH} 0.5 \mathrm{M}$ ). Posteriormente se agregaron las soluciones de las sales $\mathrm{LaCl}_{3}$ y $\mathrm{CaCl}_{2}$ en una relación volumétrica de 8:2. De acuerdo a los termogramas, se determinó que el pico correspondiente a la trasformación cristalina del gel amorfo inicia por debajo de los $750^{\circ} \mathrm{C}$. De acuerdo a estos resultados, la cristalización del gel coprecipitado se realizó mediante tratamiento térmico a $900^{\circ} \mathrm{C}$ por 3 horas, en atmósfera de aire. Los polvos obtenidos fueron compactados mediante prensado isostático en frío a $200 \mathrm{MPa}$ y las pastillas fueron sinterizadas a temperaturas en el rango de $1300-1500^{\circ} \mathrm{C}$ por intervalos de tiempo de 1, 3, 5 horas en atmósfera de aire. Las densidades máximas alcanzadas para las soluciones sólidas de $\mathrm{Ca}^{2+}$ fueron mayores al 95\% de la densidad teórica del compuesto cromita de lantano.
\end{abstract}

Palabras Claves: Soluciones Sólidas de Cromita de lantano, Coprecipitación, Medio Alcalino, Polvos Namométricos, Sinterización.

\section{Synthesis and Sintering of Lanthanum Chromite Solid Solutions Prepared by the Alkaline Coprecipitation Method.}

In the present work, three different solid solutions of lanthanum chromite, $\mathrm{La}_{0.8} \mathrm{Ca}_{0.2} \mathrm{Cr}_{1-\mathrm{x}} \mathrm{Al}_{\mathrm{x}} \mathrm{O}_{3}$, where $\mathrm{Al}=5 \mathrm{y} 10 \%$ molar; and $\mathrm{LaCr}_{0.9} \mathrm{Al}_{0.1} \mathrm{O}_{3^{\prime}}$, were prepared by the alkaline coprecipitation method. The preparation of the precursor gel was carried out by mixing solutions of $\mathrm{Cr}\left(\mathrm{NO}_{3}\right)_{3}$ and $\mathrm{AlCl}_{3}$ at two volumetric ratios (9.5:0.5 and 9:1). The $\mathrm{pH}$ of the main solution was adjusted to a value 13 by adding a $0.5 \mathrm{M} \mathrm{NaOH}$ solution. The admixture of $\mathrm{LaCl}_{3}$ and $\mathrm{CaCl}_{2}$ was then added to the latter solution, resulting in the coprecipitation of a precursor gel. Thermal analysis of the precursor gels showed that the crystallization peak of the amorphous gel occurred below $750^{\circ} \mathrm{C}$. Thus, crystallization of the gel was carried out at $900^{\circ} \mathrm{C}$ for 3 hours in air. Pellets were prepared by cold isostatic pressing at $200 \mathrm{MPa}$, and sintering was carried out at different temperatures between 1300$1500^{\circ} \mathrm{C}$ for reaction intervals between 1-5 hours in air. The maximum apparent density achieved on each of the lanthanum chromite solid solutions doped with Ca was $95 \%$ of the theoretical density.

Keywords: Lanthanum Chromite Solid Solutions, Coprecipitation, Alkaline Media, Nanometric Powders, Sintering.

\section{INTRODUCCIÓN}

La cromita de lantano y sus soluciones sólidas con $\mathrm{Ca}^{2+}$, $\mathrm{Al}^{3+}$ son materiales empleados como conductores electrónicos. Actualmente, estos materiales son usados como interconectores en las celdas de combustible de óxido sólido (SOFC's) (1). La cromita de lantano tiene una estructura tipo perovskita y propiedades que la hacen atractiva para su aplicación en equipos de generación de energía eléctrica (SOFC's) tales como: i) estabilidad química a elevadas temperaturas $\left(1000^{\circ} \mathrm{C}\right)$ en atmósferas oxidantes y reductoras, ii) coeficiente de expansión térmica $\left(9.5 \times 10^{-6} /{ }^{\circ} \mathrm{C}\right.$ de 25 a $\left.1000^{\circ} \mathrm{C}\right)$ similar al de los demás materiales que componen las celdas de combustible de óxido sólido, principalmente al compuesto de $\mathrm{Zr}_{2} \mathrm{O}$ estabilizada con $9 \% \mathrm{~mol} \mathrm{Y}_{2} \mathrm{O}_{3}$ que es usado como electrolito sólido (2), iii) además posee una alta conductividad electrónica.

Por otro lado, la cromita de lantano pura presenta un problema relacionado con su sinterización en atmósfera de aire, puesto que en estas condiciones es difícil obtener una elevada densificación en pastillas preparadas con polvos de tamaño mayor a $1 \mu \mathrm{m}$. El problema está relacionado con la evapora- ción de cromo a temperaturas superiores a $1000^{\circ} \mathrm{C}$, debido a que este elemento presenta una elevada presión parcial de vapor (3). Durante la sinterización de los polvos de cromita de lantano se forman gases de $\mathrm{CrO}_{3}$, lo que causa una deficiencia parcial de $\mathrm{Cr}$ en la composición nominal del compuesto $\mathrm{LaCrO}_{3}$. Además, la descomposición también afecta significativamente el proceso de densificación de las partículas (4).

En la actualidad, existen diversas rutas convencionales y químicas para la obtención de soluciones sólidas de cromita de lantano, dentro de las más empleadas se encuentran: i) reacción en estado sólido (5), ii) sol-gel (6,7), iii) método de Pechini (8). En general, los procesos de síntesis química mencionados (ii, iii) se han empleado para procesar polvos de cromita de lantano del tipo $\mathrm{La}_{0.9} \mathrm{Sr}_{0.1} \mathrm{Cr}_{0.95} \mathrm{Al}_{0.02} \mathrm{Co}_{0.02} \mathrm{O}_{3}$. La etapa preliminar del procesamiento de este material involucró la formación de un gel de cadena polimérica en el cual fueron incorporados de manera homogénea los iones metálicos de $\mathrm{La}^{3+}, \mathrm{Sr}^{2+}, \mathrm{Cr}^{3+}$, $\mathrm{Al}^{3+} \mathrm{y} \mathrm{Co}^{3+}$. Una vez mezcladas en proporción estequiométrica las soluciones de los diferente iones metálicos, y de acuerdo 
a la relación molar equivalente de los iones, se adicionó la cantidad correspondiente de acido cítrico y etilenglicol, compuestos orgánicos que se emplearon como precursores del gel. Posteriormente, la solución se calentó a $100^{\circ} \mathrm{C}$ durante 2 horas. En estas condiciones se formó un gel viscoso, simultáneamente los iones metálicos fueron incorporados en la red polimérica del gel (8). En general, los métodos de sol-gel y Pechini se han empleado para producir polvos finos, uniformes en tamaño y composición, sin embargo, durante la etapa de cristalización del gel precursor se requiere de temperaturas elevadas, además de que se pueden generar grandes cantidades de subproductos de reacción contaminantes debido a la presencia de los compuestos orgánicos (9).

Por otra parte, otro método alterno empleado para preparar compuestos de cromita de lantano sustituidos parcialmente en la posición $\mathrm{A}$ y $\mathrm{B}$ de la estructura $\mathrm{ABO}_{3^{\prime}}$ es el proceso de coprecipitación en medio alcalino, puesto que mediante este método se pueden obtener geles complejos constituidos por más de dos iones (9). Hasta la fecha, compuestos de cromita de lantano del tipo $\mathrm{La}_{0.7} \mathrm{Ca}_{0.3} \mathrm{Cr}_{0.95} \mathrm{Co}_{0.05} \mathrm{O}_{3}$ se han preparado mediante este proceso, involucrando la mezcla de soluciones de sales metálicas (nitratos) de $\mathrm{La}^{3+}, \mathrm{Ca}^{2+}, \mathrm{Cr}^{3+}$ y $\mathrm{Co}^{3+}$, los cuales fueron coprecipitados mediante la adición de un agente tal como el ácido oxálico $\left(\mathrm{H}_{2} \mathrm{C}_{2} \mathrm{O}_{4} \cdot 2 \mathrm{H}_{2} \mathrm{O}\right)$ con un exceso del 50 $\%$ con respecto a la relación estequiométrica de los oxalatos $\left[\mathrm{La}_{2}\left(\mathrm{C}_{2} \mathrm{O}_{4}\right)_{3^{\prime}} \mathrm{CaC}_{2} \mathrm{O}_{4}, \mathrm{CoC}_{2} \mathrm{O}_{4}\right.$ y $\left.\mathrm{Cr}_{2}\left(\mathrm{C}_{2} \mathrm{O}_{4}\right)_{3}\right]$ (9). La coprecipitación de los oxalatos se realizó a un pH de 7, el cual se obtuvo con la adición de una solución de hidróxido de amonio. El gel coprecipitado fue calcinado a 600 y $850^{\circ} \mathrm{C}$ durante 11 horas para la obtención del polvo con estructura de tipo perovskita. Esta ruta presenta la ventaja de limitar la producción de compuestos contaminantes durante la etapa de calcinado del gel precursor (9).

En el presente trabajo se plantea la obtención de polvos de cromita de lantano dopados en la posición A con la adición de $\mathrm{Ca}^{2+}$ y en la posición $\mathrm{B}$ con $\mathrm{Al}^{3+}$, mediante la técnica de coprecipitación de hidróxidos complejos en medio alcalino, empleando una base fuerte $(\mathrm{NaOH})$ a valores de $\mathrm{pH}$ por arriba de 10. Hasta la fecha mediante esta ruta solo se ha preparado el compuesto puro de $\mathrm{LaCrO}_{3}$ (10), por lo que no existe reportada en la literatura evidencia relacionada con la factibilidad de preparación de cromitas de lantano sustituidas parcialmente en las posiciones $\mathrm{A}$ y $\mathrm{B}$ de la estructura $\mathrm{ABO}_{3}$ por esta ruta. La principal ventaja de procesar un gel hidróxido complejo radica en la disminución del contenido de fases secundarias generadas durante el proceso de cristalización, principalmente $\mathrm{La}\left(\mathrm{CO}_{3}\right) \mathrm{OH}$, así como la baja temperatura requerida para transformación del hidróxido complejo en la fase cristalina del tipo perovskita (9).

\section{DESARROLLO EXPERIMENTAL.}

2.1 Síntesis de soluciones sólidas de cromita de lantano del tipo: $\mathrm{La}_{0.8} \mathrm{Ca}_{0.2} \mathrm{Cr}_{1-\mathrm{x}} \mathrm{Al}_{\mathrm{x}} \mathrm{O}_{3}$.

La etapa preliminar consistió en la preparación de un gel precursor para la obtención de los polvos de cromita de lantano, con fórmula química $\mathrm{La}_{0.8} \mathrm{Ca}_{0.2} \mathrm{Cr}_{0.9} \mathrm{Al}_{0.1} \mathrm{O}_{3}, \mathrm{La}_{0.8} \mathrm{Ca}_{0.2} \mathrm{Cr}_{0.95} \mathrm{Al}_{0.05} \mathrm{O}_{3}$ y $\mathrm{LaCr}_{0.9} \mathrm{Al}_{0.1} \mathrm{O}_{3}$; dichos geles fueron obtenidos mediante el método de coprecipitación en medio alcalino (10). Las soluciones sólidas de cromita de lantano substituidas con Ca fueron seleccionadas con el propósito de obtener pastillas con una densidad real por arriba del $95 \%$, puesto que la presencia de Ca en una concentración de hasta $20 \%$ molar, favorece la sinterización en presencia de fases liquidas transitorias (1113). En el caso particular de la substitución parcial de $\mathrm{Cr}^{3+}$ por $\mathrm{Al}^{3+}$, ésta se efectuó con el objetivo de controlar el coeficiente de expansión térmica del compuesto, puesto que el ión $\mathrm{Al}$ permite obtener un coeficiente de expansión térmica similar al del electrolito sólido $\left(10.3 \times 10^{-6} /{ }^{\circ} \mathrm{C}\right.$ de 25 a $\left.1000^{\circ} \mathrm{C}\right)$ cuando se incorpora en una cantidad menor al $10 \%$ molar; por arriba de esta concentración se disminuye considerablemente la conductividad eléctrica del compuesto (14). En el caso particular de la tercera composición, se optó por sustituir el $\mathrm{Cr}^{3+}$ parcialmente con $\mathrm{Al}^{3+}$, para evaluar la posibilidad de obtener una buena densificación en función únicamente del tamaño del polvo del gel precursor en ausencia de $\mathrm{Ca}^{2+}$.

Inicialmente, se prepararon soluciones de $\mathrm{Cr}\left(\mathrm{NO}_{3}\right)_{3}$ (99.99\%, Aldrich), $\mathrm{LaCl}_{3}$ (99.999\%, Aldrich), $\mathrm{CaCl}_{2}$ (99.99\%, Aldrich), $\mathrm{AlCl}_{3}$ (99.99\%, Aldrich), con una concentración 0.05 $\mathrm{M}$. Cabe hacer mención que todas las soluciones empleadas y el gel fueron preparadas en una caja de guantes con una atmósfera de $\mathrm{N}_{2}$, para evitar la hidratación de los cloruros y la carbonatación del gel hidróxido complejo, la coprecipitación del gel hidróxido complejo se realizó a temperatura ambiente $\left(25^{\circ} \mathrm{C}\right)$.

Los cationes metálicos de $\mathrm{Cr}^{3+} \mathrm{y} \mathrm{Al}^{3+}$ normalmente precipitan en condiciones ácidas y básicas, debido a su carácter anfótero, por lo tanto, inicialmente se preparó una mezcla $(500 \mathrm{ml})$ con las soluciones de $\mathrm{Cr}\left(\mathrm{NO}_{3}\right)_{3}$ y $\mathrm{AlCl}_{3^{\prime}}$ en dos relaciones en volumen (9.5:0.5 y 9:1) las cuales corresponden a la relación estequiométrica de la posición $\mathrm{B}$ de la estructura $\mathrm{ABO}_{3}$, adicionándose posteriormente la solución de $\mathrm{NaOH}$ $(350 \mathrm{ml})$ de concentración $0.5 \mathrm{M}$, durante esta etapa se monitoreó continuamente la variación del pH de la solución inicial. Durante la incorporación de la solución alcalina se formaron los hidróxidos correspondientes de $\mathrm{Cr}(\mathrm{OH})_{3}$ y $\mathrm{Al}(\mathrm{OH})_{3}$ a un $\mathrm{pH}$ de 6, y estos compuestos se disolvieron cuando el $\mathrm{pH}$ de la mezcla de soluciones alcanzó un valor de 13.2 una vez adicionado el medio de coprecipitación alcalino. Finalmente, se agregó una tercera mezcla $(500 \mathrm{ml})$ de las soluciones de $\mathrm{LaCl}_{3}$ y $\mathrm{CaCl}_{2}$ mezcladas en una relación en volumen de 8:2, de carácter ácido $(\mathrm{pH}=5.01)$. En cuanto se adicionó la tercera solución, el pH de la solución disminuyó hasta un valor de 12.5 y simultáneamente se formó un precipitado de color verde opaco. Luego la solución se dejó sedimentar durante 1 hora en atmósfera de $\mathrm{N}_{2}$ y posteriormente se centrifugó a 3000 rpm durante 9 minutos para separar el gel precipitado de la solución remanente (10).

El gel precursor fue secado a $110^{\circ} \mathrm{C}$ durante 24 horas, y después el gel seco se molturó en un mortero y se cristalizó mediante tratamiento térmico a $900^{\circ} \mathrm{C}$ en atmósfera de aire durante 3 horas. El calentamiento del horno se realizó a una velocidad de $10^{\circ} \mathrm{C} / \mathrm{min}$. El polvo tratado se molturó durante 5 horas en un molino de bolas, usando un medio de molienda de circona e isopropanol para activar la dispersión de las partículas. Finalmente el polvo fue secado a $50^{\circ} \mathrm{C}$ durante 12 horas.

\subsection{Sinterización de soluciones sólidas de cromita de lantano dopadas con $\mathrm{Ca}^{2+} \mathrm{y} \mathrm{Al}^{3+}$.}


Una vez obtenidos los polvos de las soluciones sólidas de cromita de lantano dopadas con $\mathrm{Ca}^{2+} \mathrm{y} \mathrm{Al}^{3+}$, se prepararon pastillas mediante prensado isostático en frío aplicando una carga nominal de compactación de $200 \mathrm{MPa}$. Posteriormente las pastillas fueron sometidas a tratamiento térmico con la finalidad de obtener cuerpos con densidades superiores al 92\% de la densidad teórica del compuesto cromita de lantano pura. Los tratamientos térmicos para llevar a cabo el sinterizado de las pastillas se realizaron a temperaturas en el rango de $1300-1500^{\circ} \mathrm{C}$ durante tiempos de reacción en el intervalo de 1 a 5 horas. Cabe mencionar que todas las muestras fueron sinterizadas en atmósfera de aire.

\subsection{Caracterización de soluciones sólidas de cromita de lan- tano dopados con $\mathrm{Ca}^{2+}$ y $\mathrm{Al}^{3+}$.}

La caracterización del gel precursor, se llevó a cabo mediante un equipo de análisis térmico diferencial (ATD) Perkin Elmer DTA-7, a una velocidad de calentamiento de $10^{\circ} \mathrm{C} /$ min, con el propósito de determinar la estabilidad térmica del gel precursor así como la temperatura de cristalización de las soluciones sólidas de cromita de lantano sustituidas con $\mathrm{Ca}^{2+} \mathrm{y} \mathrm{Al}^{3+}$. El análisis de las fases cristalinas presentes en los polvos calcinados se realizó mediante la técnica de difracción de rayos X (DRX-Rigaku-Rotaflex) utilizando $40 \mathrm{kV}$ y $100 \mathrm{~mA}$, con una radiación $\mathrm{Cu} \mathrm{K} \alpha$ monocromada con grafito, en un rango de ángulo de barrido de $15-55^{\circ}$. Aspectos del tamaño y forma de los polvos de las soluciones sólidas de cromita de lantano tratados térmicamente se determinaron mediante microscopía electrónica de barrido (MEB, Phillips XL 30SEM) equipado con una unidad de microanálisis de rayos $\mathrm{X}$ (EDAX) mediante la cual se realizaron análisis cuantitativos para determinar el contenido de cada elemento en los polvos tratados térmicamente y las pastillas sinterizadas. Otros aspectos puntuales se caracterizaron mediante la microscopía electrónica de transmisión, dichas micrográfias se obtuvieron en un microscopio Phillips CM200. La densidad real de los cuerpos sinterizados se midió mediante picnometría de helio (Quantachrome PYC300E), a una presión de gas de $0.117 \mathrm{MPa}$.

\section{RESULTADOS Y DISCUSIÓN.}

\subsection{Síntesis de soluciones sólidas de cromita de lantano del tipo $\mathrm{La}_{0.8} \mathrm{Ca}_{0.2} \mathrm{Cr}_{1-\mathrm{x}} \mathrm{Al}_{\mathrm{x}} \mathrm{O}_{3}$.}

En la figura 1 se muestra el análisis térmico diferencial (ATD), correspondiente a los tres geles precursores preparados para la obtención de las soluciones sólidas de cromita de lantano: (a) $\mathrm{LaCr}_{0.9} \mathrm{Al}_{0.1} \mathrm{O}_{3}$; (b) $\mathrm{La}_{0.8} \mathrm{Ca}_{0.2} \mathrm{Cr}_{0.9} \mathrm{Al}_{0.1} \mathrm{O}_{3}$; (c) $\mathrm{La}_{0.8} \mathrm{Ca}_{0.2}$ $\mathrm{Cr}_{0.95} \mathrm{Al}_{0.05} \mathrm{O}_{3}$. En general en los tres casos se observa un pico endotérmico localizado aproximadamente a $127^{\circ} \mathrm{C}$, debido a la pérdida del agua absorbida físicamente y la enlazada químicamente en los geles precursores. Por otro lado, en el termograma del compuesto $\mathrm{La}_{0.8} \mathrm{Ca}_{0.2} \mathrm{Cr}_{0.95} \mathrm{Al}_{0.05} \mathrm{O}_{3}$ (figura 1c) se observa un pico de carácter exotérmico a $470^{\circ} \mathrm{C}$. De acuerdo a resultados determinados previamente para la cristalización de geles precursores de soluciones sólidas de cromita de lantano (15), se determinó que este pico corresponde a la formación del compuesto cromato de lantano $\left(\mathrm{LaCrO}_{4}\right)$, el cual es una fase intermedia que se forma durante la obtención de la fase

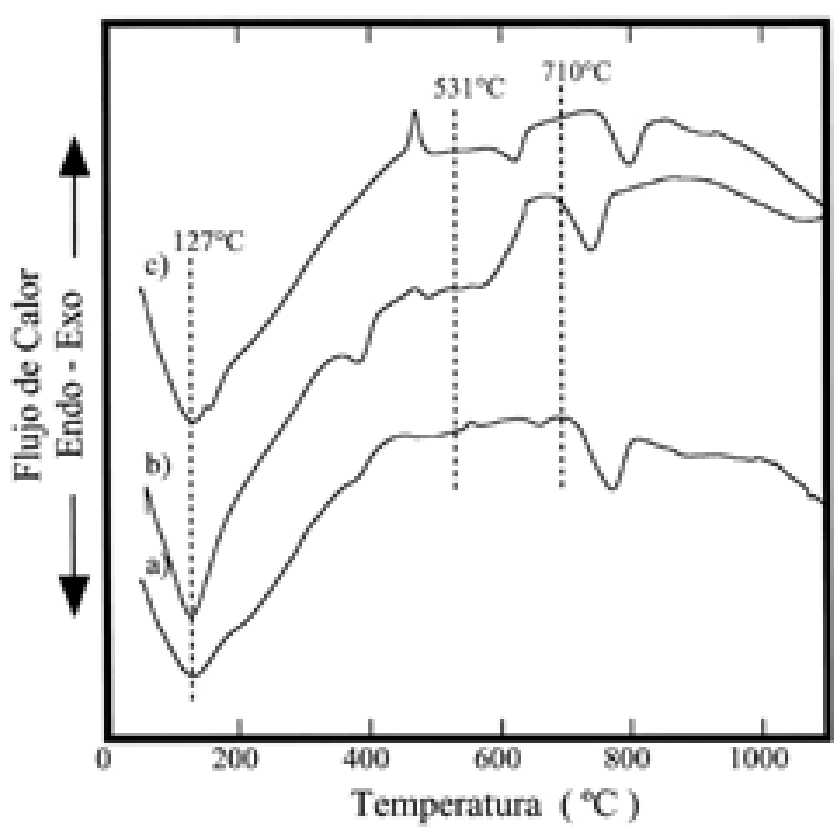

Figura 1. Análisis térmico diferencial (ATD), de las soluciones sólidas de, (a) $\mathrm{LaCr}_{0.9} \mathrm{Al}_{0.1} \mathrm{O}_{3} ;$ (b) $\mathrm{La}_{0.8} \mathrm{Ca}_{0.2} \mathrm{Cr}_{0.9} \mathrm{Al}_{0.1} \mathrm{O}_{3}$; (c) $\mathrm{La}_{0.8} \mathrm{Ca}_{0.2} \mathrm{Cr}_{0.95} \mathrm{Al}_{0.05} \mathrm{O}_{3}$. $\mathrm{El}$ análisis se efectuó a una velocidad de calentamiento de $10^{\circ} \mathrm{C} / \mathrm{min}$.

cromita de lantano. A temperaturas superiores a $710^{\circ} \mathrm{C}$ se observó la formación de un pico endotérmico, el cual corresponde a la transformación amorfo-cristalina del compuesto de cromita de lantano para los tres geles precursores. Además, este pico presenta un desplazamiento significativo en los termogramas, el cual se atribuye que está asociado principalmente al grado de incorporación de los cationes dopantes dentro de las posiciones A y B de la estructura cristalina de la cromita de lantano $\left(\mathrm{ABO}_{3}\right)$. De acuerdo a los resultados de ATD, se determinó que la calcinación de los geles precursores se efectuara a una temperatura de $900^{\circ} \mathrm{C}$. Después de realizar una serie de pruebas preliminares se encontró que el tiempo requerido para cristalizar completamente el gel precursor a esta temperatura era de 3 horas.

Una vez que los geles precursores fueron tratados térmicamente se caracterizaron mediante difracción de rayos $X$. Los patrones de difracción correspondientes a los polvos de $\mathrm{LaCr}_{0.9} \mathrm{Al}_{0.1} \mathrm{O}_{3^{\prime}} \mathrm{La}_{0.8} \mathrm{Ca}_{0.2} \mathrm{Cr}_{0.9} \mathrm{Al}_{0.1} \mathrm{O}_{3^{\prime}}$ y $\mathrm{La}_{0.8} \mathrm{Ca}_{0.2} \mathrm{Cr}_{0.95} \mathrm{Al}_{0.05} \mathrm{O}_{3}$ obtenidos mediante la ruta convencional se muestran en la figura 2. En los patrones de difracción se observa que en las soluciones sólidas de cromita de lantano dopadas con $\mathrm{Ca}^{2+} \mathrm{y} \mathrm{Al}^{3+}$ en las posiciones A y $\mathrm{B}$ respectivamente, presentan una mínima contaminación con subproductos de reacción principalmente trazas del compuesto $\mathrm{CaCrO}_{4}$ (JCPDS 08-0458). La estructura de los polvos obtenidos fue indexada con la del compuesto puro de $\mathrm{LaCrO}_{3}$ (carta JCPDS 33-701) con estructura ortorrómbica. Por otro lado, se puede observar que los picos de difracción presentan un ligero desplazamiento hacia ángulos menores con respecto a la posición de los picos de difracción del compuesto con estructura ortorrómbica $\mathrm{LaCrO}_{3}$. Este fenómeno está asociado con la incorporación de los iones de $\mathrm{Ca}^{2+}$ $\mathrm{y} \mathrm{Al}^{3+}$ que se ubican en las posiciones $\mathrm{A}$ y $\mathrm{B}$, y disminuyen los parámetros reticulares de la celda debido a que tienen menor radio atómico $\left(\mathrm{Ca}^{2+}=0.99 \AA, \mathrm{Al}^{3+}=0.50 \AA\right)$ en comparación con el $\mathrm{La}^{3+}(1.15 \AA ̊)$ y $\mathrm{Cr}^{3+}(0.52 \AA)$. Esta suposición concuerda con los 


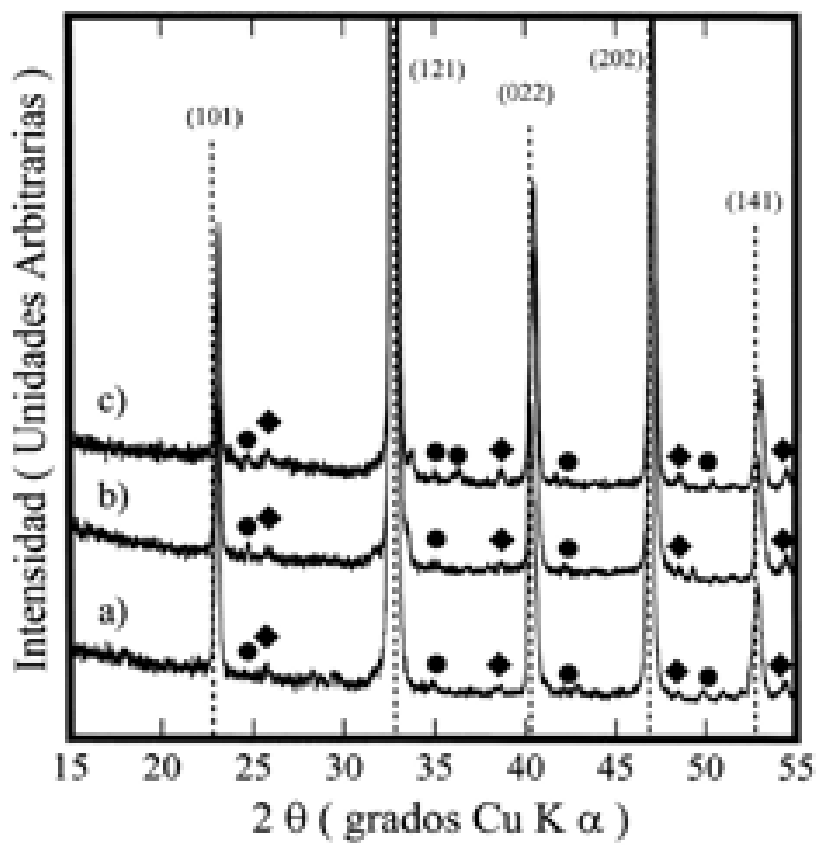

Figura 2. Patrón de difracción de rayos $\mathrm{X}$ de polvos de cromita de lantano dopados con $\mathrm{Ca}$ y $\mathrm{Al}$, tratados térmicamente a $900^{\circ} \mathrm{C}$ durante 3 horas. (a) $\mathrm{LaCr}_{0.9} \mathrm{Al}_{0.1} \mathrm{O}_{3}$; (b) $\mathrm{La}_{0.8} \mathrm{Ca}_{0.2} \mathrm{Cr}_{0.9} \mathrm{Al}_{0.1} \mathrm{O}_{3}$; (c) $\mathrm{La}_{0.8} \mathrm{Ca}_{0.2} \mathrm{Cr}_{0.95} \mathrm{Al}_{0.0}$ ${ }_{5} \mathrm{O}_{3}$. Las líneas punteadas representan los planos de mayor intensidad y el símbolo $(\diamond)$ representa los picos de baja intensidad de $\mathrm{LaCrO}_{3}$ con estructura ortorrómbica (carta JDCPS 33-701). (•) Picos correspondientes al compuesto de $\mathrm{CaCrO}_{4}$.

análisis cuantitativos realizados por EDS, los cuales muestran que los contenidos de los elementos $\mathrm{La}^{3+}, \mathrm{Ca}^{2+}, \mathrm{Cr}^{3+} \mathrm{y} \mathrm{Al}^{2+}$ son similares a las relaciones estequiométricas nominales de las soluciones sólidas de cromita de lantano (Tabla 1). De acuerdo a los resultados anteriores, se concluye que es posible obtener las soluciones sólidas de cromita de lantano dopadas con $\mathrm{Ca}^{2+}$ y $\mathrm{Al}^{3+}$ mediante la técnica de coprecipitación en medio alcalino y un calcinado posterior a $900^{\circ} \mathrm{C}$ durante 3 horas.

TABLA 1. CONTENIDO DE LOS ELEMENTOS $\mathrm{La}^{3+}, \mathrm{Ca}^{2+}, \mathrm{Cr}^{3+} \mathrm{y} \mathrm{Al}^{3+}$

DETERMINADOS MEDIANTE ESPECTROSCOPIA DE ENERGÍA DISPERSIVA DE RAYOS $X$, EN LOS POLVOS Y EN LAS PASTILLAS SINTERIZADAS CORRESPONDIENTES A LAS SOLUCIONES SÓLIDAS DE CROMITA DE LANTANO.

\begin{tabular}{|c|c|c|c|c|c|c|c|}
\hline \multirow{2}{*}{ Muestra } & \multicolumn{2}{|c|}{$\begin{array}{l}\text { Condiciones de } \\
\text { Sinterización }\end{array}$} & \multicolumn{4}{|c|}{$\begin{array}{c}\text { Composición } \\
\text { de los elementos (\% atómico ) }\end{array}$} & \multirow{2}{*}{$\begin{array}{c}\text { Relación } \\
\text { estequiométrica } \\
\text { La:Ca:Cr:Al }\end{array}$} \\
\hline & \begin{tabular}{|c|}
$\begin{array}{c}\text { Temperatura } \\
\left({ }^{\circ} \mathrm{C}\right)\end{array}$ \\
\end{tabular} & $\begin{array}{l}\text { Tiempo } \\
\text { (horas) }\end{array}$ & $\mathrm{La}$ & $\mathrm{Ca}$ & $\mathrm{Cr}$ & $\mathrm{Al}$ & \\
\hline LCA (Polvo inicial) $)^{\star}$ & - & - & $50.0(2)$ & - & $44.9(2)$ & 5.1(2) & 1.0:00.0:0.9:0.1 \\
\hline LCCA5 (Polvo inicial) $)^{*}$ & - & - & $39.9(4)$ & $10.1(4)$ & $47.9(4)$ & $2.1(4)$ & 0.8:0.2:0.95:0.05 \\
\hline LCCA10 (Polvo inicial)* & - & - & $40.5(7)$ & $9.5(7)$ & $44.9(7)$ & $5.1(7)$ & \begin{tabular}{|l|} 
0.8:0.2:0.9:0.1 \\
\end{tabular} \\
\hline LCA-1 & 1500 & 5 & $50.3(2)$ & - & $44.1(2)$ & $5.6(2)$ & \begin{tabular}{|l|}
$10: 00.0: 0.9: 0: 0.1$ \\
\end{tabular} \\
\hline LCCA5-1 & 1400 & 5 & $40.2(5)$ & $9.8(5)$ & $47.2(5)$ & $2.8(5)$ & 0.8:0.2:0.95:0.05 \\
\hline LCCA5-2 & 1500 & 5 & $40.3(5)$ & $9.7(5)$ & $43.9(5)$ & $6.1(5)$ & 0.8:0.2:0.95:0.05 \\
\hline LCCA10-1 & 1400 & 5 & $40.1(7)$ & $9.9(7)$ & $44.9(7)$ & $5.1(7)$ & \begin{tabular}{|l|} 
0.8:0.2:0.9:0.1 \\
\end{tabular} \\
\hline LCCA10-2 & 1500 & 5 & $40.4(7)$ & $9.6(7)$ & $43.5(7)$ & $6.5(7)$ & \begin{tabular}{|l|l|} 
0.8:0.2:0.9:0.1 \\
\end{tabular} \\
\hline
\end{tabular}

* Nota: Composición de los polvos calcinados a una temperatura de $900^{\circ} \mathrm{C}$ durante 3 horas, en una atmósfera de aire.

${ }^{* \star}$ El paréntesis denota el porcentaje de error estimado en base a 10 mediciones realizadas para cada elemento.

En la figura 3 se muestran las micrográfias de los polvos de las soluciones sólidas de cromita de lantano obtenidos mediante tratamiento térmico a $900^{\circ} \mathrm{C}$. En la figura 3a correspondiente a la solución sólida dopada con $10 \%$ molar de $\mathrm{Al}^{3+}$.
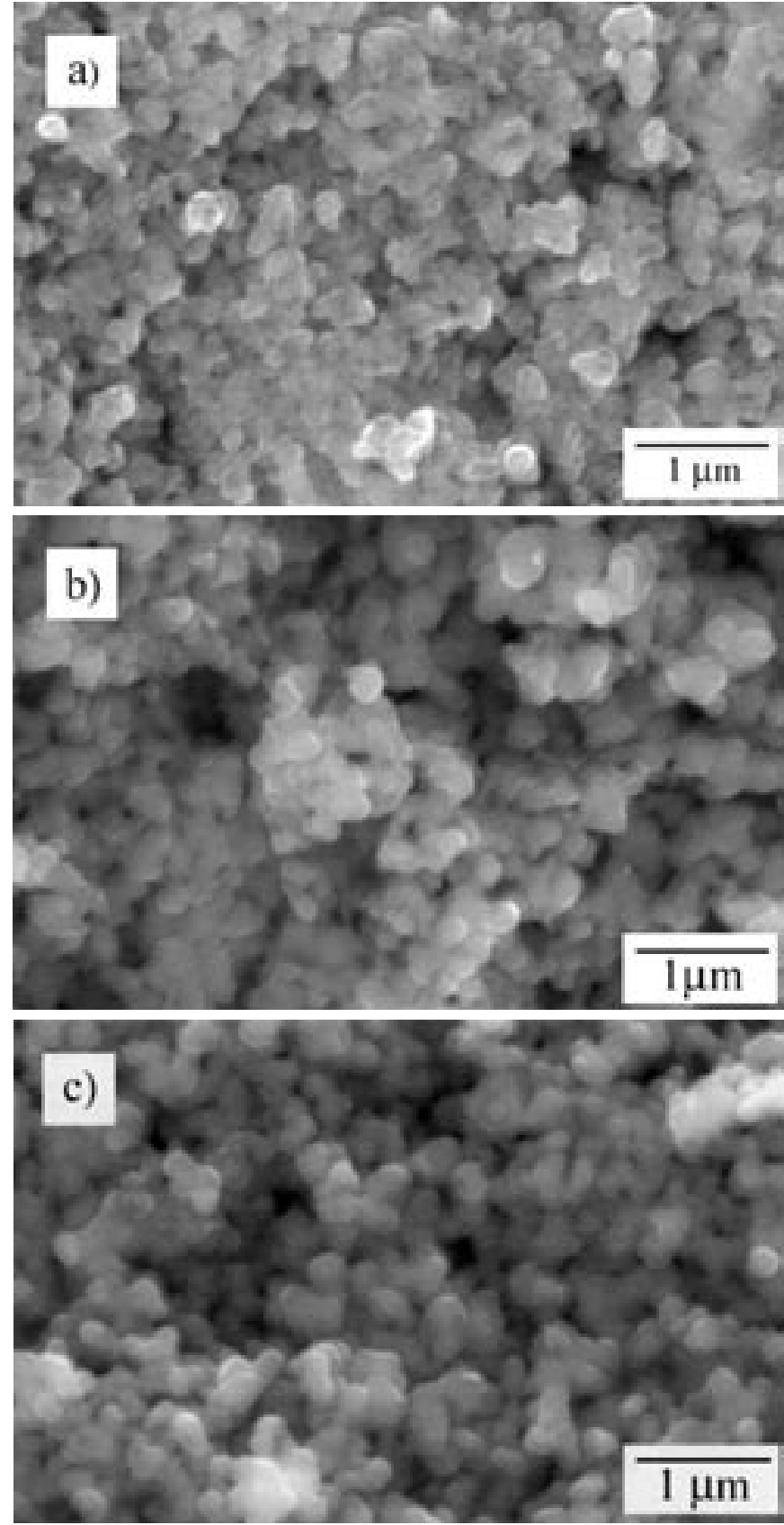

Figura 3. Micrografías obtenidas mediante MEB de polvos de cromita de lantano dopados con Ca y Al, (a) $\mathrm{LaCr}_{0.9} \mathrm{Al}_{0.1} \mathrm{O}_{3}$; (b) $\mathrm{La}_{0.8} \mathrm{Ca}_{0.2} \mathrm{Cr}_{0.9} \mathrm{Al}_{0.1} \mathrm{O}_{3^{\prime}}$ (c) $\mathrm{La}_{0.8} \mathrm{Ca}_{0.2} \mathrm{Cr}_{0.95} \mathrm{Al}_{0.05} \mathrm{O}_{3^{\prime}}$ preparados mediante síntesis por coprecipitación y tratados a $900^{\circ} \mathrm{C}$ durante 3 horas.

Se puede observar una aglomerización significativa de los polvos, aún después de que estos fueron molidos durante 5 horas, y las partículas presentan formas homogéneas con superficies curvas. En contraste, los polvos de soluciones sólidas del tipo: $\mathrm{La}_{0.8} \mathrm{Ca}_{0.2} \mathrm{Cr}_{0.95} \mathrm{Al}_{0.05} \mathrm{O}_{3}$ (figura 3b), $\mathrm{La}_{0.8} \mathrm{Ca}_{0.2} \mathrm{Cr}_{0.9} \mathrm{Al}_{0.1} \mathrm{O}_{3}$ (figura $3 c)$, consisten en partículas de forma regular y homogénea en tamaño, presentando superficies bien definidas. Por otra parte, las micrografías revelan que existen diferencias en el tamaño de las partículas para cada una de las soluciones sólidas preparadas, es decir, presentan un ligero incremento en el tamaño de las partículas con el aumento del contenido de dopante. En el caso de la solución sólida dopada con $10 \%$ 
molar de $\mathrm{Al}^{3+}$ en la posición B (figura 3c), se observa que los polvos obtenidos tienen tamaños de partícula menores a 0.2 $\mu \mathrm{m}$. En contraste, las soluciones sólidas dopadas con $\mathrm{Ca}^{2+} \mathrm{y}$ $\mathrm{Al}^{3+}$ (figuras $3 \mathrm{a}$ y $3 \mathrm{~b}$ ) revelan que los polvos presentan un tamaño de partícula mayor (0.3-0.4 $\mu \mathrm{m})$. De la misma manera, se observó que con la adición de $\mathrm{Ca}^{2+}$ como dopante en la posición A de la estructura, la morfología de los polvos obtenidos se torna más regular. Las micrografías de los polvos muestran que un incremento en el tamaño de las partículas $(0.35 \mu \mathrm{m})$ de la solución sólida del tipo $\mathrm{La}_{08} \mathrm{Ca}_{02} \mathrm{Cr}_{1-x} \mathrm{Al}_{x} \mathrm{O}_{3}$, se puede activar con el aumento en el contenido de $\mathrm{Al}^{3+}$ a un valor de $10 \%$ molar de $\mathrm{Al}^{3+}$. Por otro lado, mediante microscopía electrónica de transmisión, se revelaron otros aspectos de la morfología de las soluciones sólidas de cromita de lantano dopadas con $\mathrm{Ca}^{2+} \mathrm{y} \mathrm{Al}^{3+}$. En la figura 4a se presenta la micrografía obtenida para la solución sólida del tipo $\mathrm{La}_{0.8} \mathrm{Ca}_{0.2} \mathrm{Cr}_{0.9} \mathrm{Al}_{0.1} \mathrm{O}_{3^{\prime}}$ la cual muestra que las partículas presentan formas regulares (esféricas) y homogéneas en tamaño $(0.25 \mu \mathrm{m})$, algunas de estas partículas se encuentran separadas. En la figura 4 b se presenta la micrografía para la solución sólida del tipo $\mathrm{La}_{0.8} \mathrm{Ca}_{0.2} \mathrm{Cr}_{0.95} \mathrm{Al}_{0}$. ${ }_{05} \mathrm{O}_{3^{\prime}}$, donde se observa que las partículas presentan una mayor aglomeración que las de la solución sólida con $10 \%$ molar de $\mathrm{Al}$, y aunque presentan formas definidas el tamaño de partícula no es homogéneo.
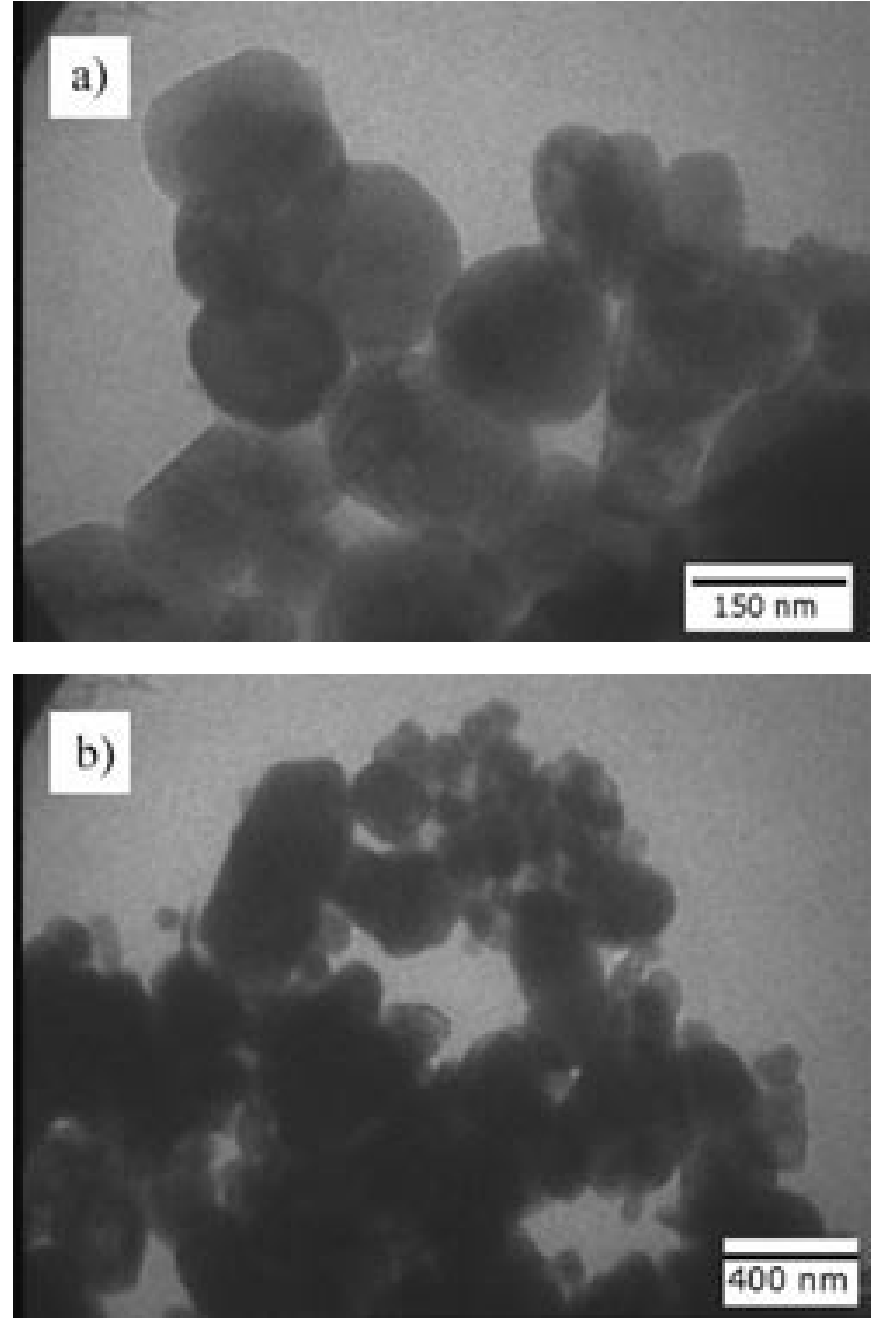

Figura 4. Micrografías microscopia electrónica de transmisión de polvos de cromita de lantano, a) $\mathrm{La}_{0.8} \mathrm{Ca}_{0.2} \mathrm{Cr}_{0.9} \mathrm{Al}_{0.1} \mathrm{O}_{3}$ y

(b) $\mathrm{La}_{0.8} \mathrm{Ca}_{0.2} \mathrm{Cr}_{0.95} \mathrm{Al}_{0.05} \mathrm{O}_{3}$

\subsection{Sinterización de polvos de cromita de lantano del tipo $\mathrm{La}_{0.8} \mathrm{Ca}_{0.2} \mathrm{Cr}_{1-\mathrm{x}} \mathrm{Al}_{\mathrm{x}} \mathrm{O}_{3}$.}

Una vez caracterizados los polvos de soluciones sólidas de cromita de lantano, se procedió a sinterizar los polvos a temperaturas en el rango de $1300-1500^{\circ} \mathrm{C}$ por diferentes intervalos de tiempo (1, 3, 5 horas), debido a que en trabajos previos bajo estas condiciones se ha logrado obtener cuerpos densificados de soluciones sólidas de cromita de lantano, con densidades superiores al $92 \%$ de la densidad real $(3,12,13)$. Los resultados correspondientes a la densidad real obtenida para cada una de las soluciones sólidas dopadas con $\mathrm{Ca}^{2+} \mathrm{y}$ $\mathrm{Al}^{3+}$, se muestran en la figura $5 \mathrm{a}$ y $5 \mathrm{~b}$. En general, se observa que las dos soluciones sólidas presentan un comportamiento similar durante la etapa de sinterizado a temperaturas bajas $\left(1300-1400^{\circ} \mathrm{C}\right)$. En estas condiciones se obtuvo un incremento paulatino de la densidad real conforme se aumenta el tiempo de sinterización. Sin embargo, a tiempos cortos de sinterizado (1 hora), la solución sólida dopada con $20 \%$ molar de $\mathrm{Ca}^{2+}$ y $5 \%$ molar de $\mathrm{Al}^{3+}$ presenta valores de densidad menores, en comparación con los de la solución sólida dopada con $20 \%$ molar de $\mathrm{Ca}^{2+}$ y $10 \%$ molar de $\mathrm{Al}^{3+}$. Sin embargo, la densidad real de dichas soluciones sólidas fue similar cuando el tiempo de sinterizado se incrementó hasta 5 horas. A la
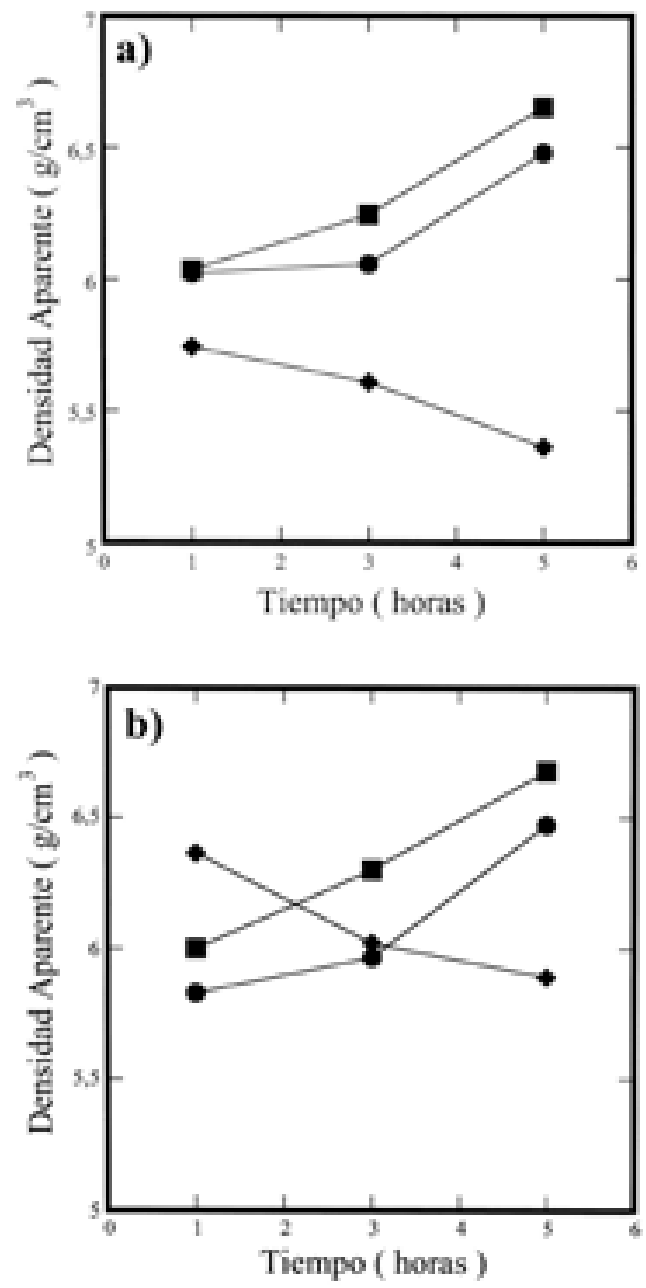

Figura 5. Variación de la densidad real con respecto al tiempo de sinterización para las soluciones sólidas del tipo. a) $\mathrm{La}_{0.8} \mathrm{Ca}_{0.2} \mathrm{Cr}_{0.9} \mathrm{Al}_{0.1} \mathrm{O}_{3}$; b) $\mathrm{La}_{0} \mathrm{Ca}_{02} \mathrm{Cr}_{095} \mathrm{Al}_{005} \mathrm{O}_{3}$, tratadas a diferentes temperaturas, $(\bullet)$ $1300^{\circ} \mathrm{C} ;(\mathbf{\square}) 1400^{\circ} \mathrm{C} ;(\diamond) 1500^{\circ} \mathrm{C}$. 
temperatura de sinterización de $1500^{\circ} \mathrm{C}$ presenta en ambos casos una disminución abrupta de la densidad real de estos compuestos, como se observa en las figuras 5 a y $5 b$, conforme se incrementa el tiempo de reacción. Esta disminución en los valores de densidad se debe a que a $1500^{\circ} \mathrm{C}$ la evaporación del elemento $\mathrm{Cr}^{3+}$ se favorece en atmósferas oxidantes, debido a su elevada presión parcial de vapor lo que causa una deficiencia parcial de $\mathrm{Cr}^{3+}$ en la composición nominal y afecta el proceso de sinterización de la cromita de lantano $(3,4,10)$. Lo anterior se confirmó en las muestras de las soluciones sólidas $\mathrm{La}_{0.8} \mathrm{Ca}_{0.2} \mathrm{Cr}_{0.95} \mathrm{Al}_{0.05} \mathrm{O}_{3}$ (muestra LCCA5-2) y $\mathrm{La}_{0.8} \mathrm{Ca}_{0.2} \mathrm{Cr}_{0.9} \mathrm{Al}_{0.1} \mathrm{O}_{3}$ (muestra LCCA10-2) sinterizadas a $1500^{\circ} \mathrm{C}$ durante 5 horas (Tabla 1), las cuales presentan una ligera disminución en el contenido de $\mathrm{Cr}^{3+}$ con respecto a la composición nominal del polvo y de las mismas pastillas tratadas a menor temperatura $\left(1400^{\circ} \mathrm{C}\right)$.

En la figura 6 se muestran los resultados de densidad real para la solución sólida de cromita de lantano dopada con el $10 \%$ molar de $\mathrm{Al}^{3+}$ en la posición $\mathrm{B}$, determinadas en las muestras sinterizadas a $1500^{\circ} \mathrm{C}$ a diferentes tiempos de reacción. En particular se observa que bajo estas condiciones de temperatura sólo se logró obtener valores de densidad en las pastillas sinterizadas del orden de $85 \%$ de la densidad teórica del compuesto $\mathrm{LaCrO}_{3}$. La baja sinterabilidad que tienen estos polvos finos en tamaño, se estima que se debe a la ausencia de $\mathrm{Ca}^{2+}$ en la composición química de la solución sólida, puesto que en presencia de $\mathrm{Ca}^{2+}$ y $\mathrm{Sr}^{2+}$ la sinterización de cromitas de lantano se favorece en atmósferas oxidantes por la formación de líquidos transitorios de bajos puntos de fusión $(11,12)$, tal como es el caso de las soluciones sólidas del tipo $\mathrm{La}_{0.8} \mathrm{Ca}_{0.2} \mathrm{Cr}_{1}$ $\mathrm{Al}_{\mathrm{x}} \mathrm{O}_{3}$ preparadas en el presente trabajo (figuras $5 \mathrm{a}$ y $5 \mathrm{~b}$ ), en las cuales los valores de densidad real alcanzados se encuentran por encima del $95 \%$ de la densidad teórica del compuesto $\mathrm{LaCrO}_{3}$.

En la figura 7 se presentan las micrografías de la microestructura de los materiales sinterizados correspondientes a las soluciones sólidas del tipo: $\mathrm{La}_{0.8} \mathrm{Ca}_{0.2} \mathrm{Cr}_{0.9} \mathrm{Al}_{0.1} \mathrm{O}_{3^{\prime}} \mathrm{La}_{0.8} \mathrm{Ca}_{0.2} \mathrm{Cr}_{0}$. ${ }_{95} \mathrm{Al}_{0.05} \mathrm{O}_{3^{\prime}}$ obtenidas a $1400^{\circ} \mathrm{C}$ durante 5 horas en atmósferas de aire. En ambos casos, la microestructura de las pastillas sinterizadas consiste en granos equiaxiales y algunos poros remanentes, los cuales se encuentran localizados en límites de grano y puntos triples. Como característica particular la muestra $\mathrm{La}_{0.8} \mathrm{Ca}_{0.2} \mathrm{Cr}_{0.9} \mathrm{Al}_{0.1} \mathrm{O}_{3}$ presenta un tamaño de grano más homogéneo con un valor promedio de $1.5 \mu \mathrm{m}$ (figura 7a). En contraste la solución sólida $\mathrm{La}_{0.8} \mathrm{Ca}_{0.2} \mathrm{Cr}_{0.95} \mathrm{Al}_{0.05} \mathrm{O}_{3^{\prime}}$ presentó un crecimiento anormal de grano. De acuerdo con las mediciones efectuadas se determinó que el tamaño promedio de los granos es de $4 \mu \mathrm{m}$. El crecimiento anormal de grano observado se estima que se debe a la gran heterogeneidad en el tamaño que presentan las partículas de cromita de lantano calcinadas (figura $4 \mathrm{~b}$ ). Por otra parte, en la figura 8 se muestra la micrografía para la solución sólida $\mathrm{LaCr}_{0.9} \mathrm{Al}_{0.1} \mathrm{O}_{3^{\prime}}$, sinterizada a $1400^{\circ} \mathrm{C}$ durante 5 horas, en la cual se observa que los granos no presentan formas definidas, puesto que el mecanismo de sinterización no se activó en estas soluciones sólidas. Lo anterior se debió a la carencia de elementos como el $\mathrm{Ca}^{2+}(8,11)$. La máxima densidad alcanzada en las pastillas tratadas a $1400^{\circ} \mathrm{C}$ por 5 horas, fue del $85 \%$ de la densidad teórica del compuesto $\mathrm{LaCrO}_{3}$. Los resultados obtenidos concuerdan con la densidad real medida para estas muestras y con resultados obtenidos previamente para este tipo de soluciones sólidas de cromita de lantano (13).

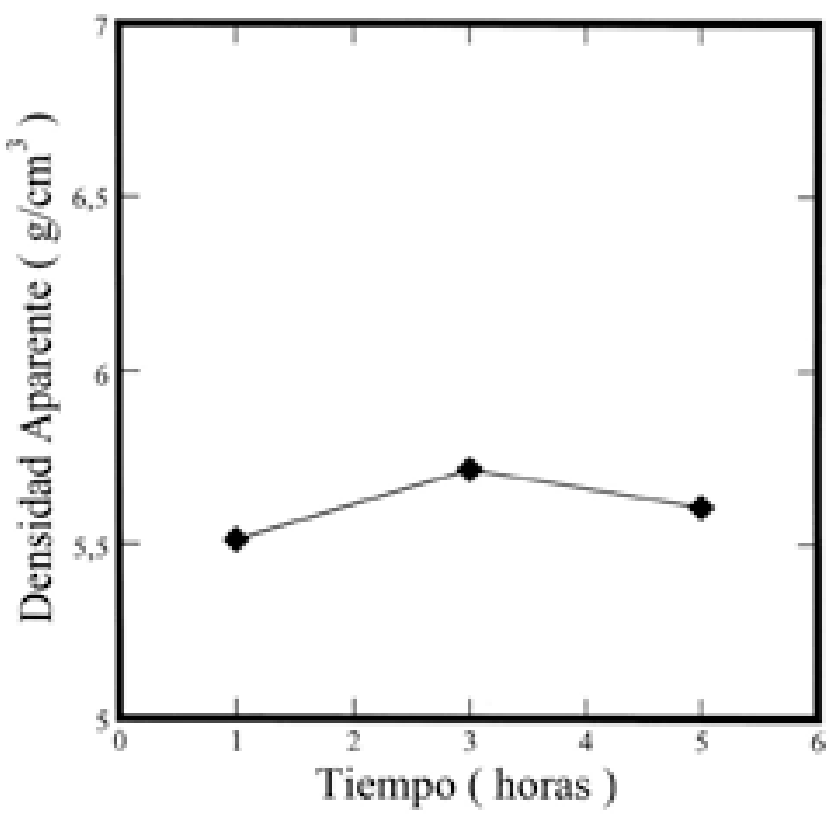

Figura 6. Variación de la densidad real con respecto al tiempo de sinterización para la solución sólida de cromita de lantano: $\mathrm{LaCr}_{0.9} \mathrm{Al}_{0.1} \mathrm{O}_{3}$ para la muestra sinterizada a $1500^{\circ} \mathrm{C}$.
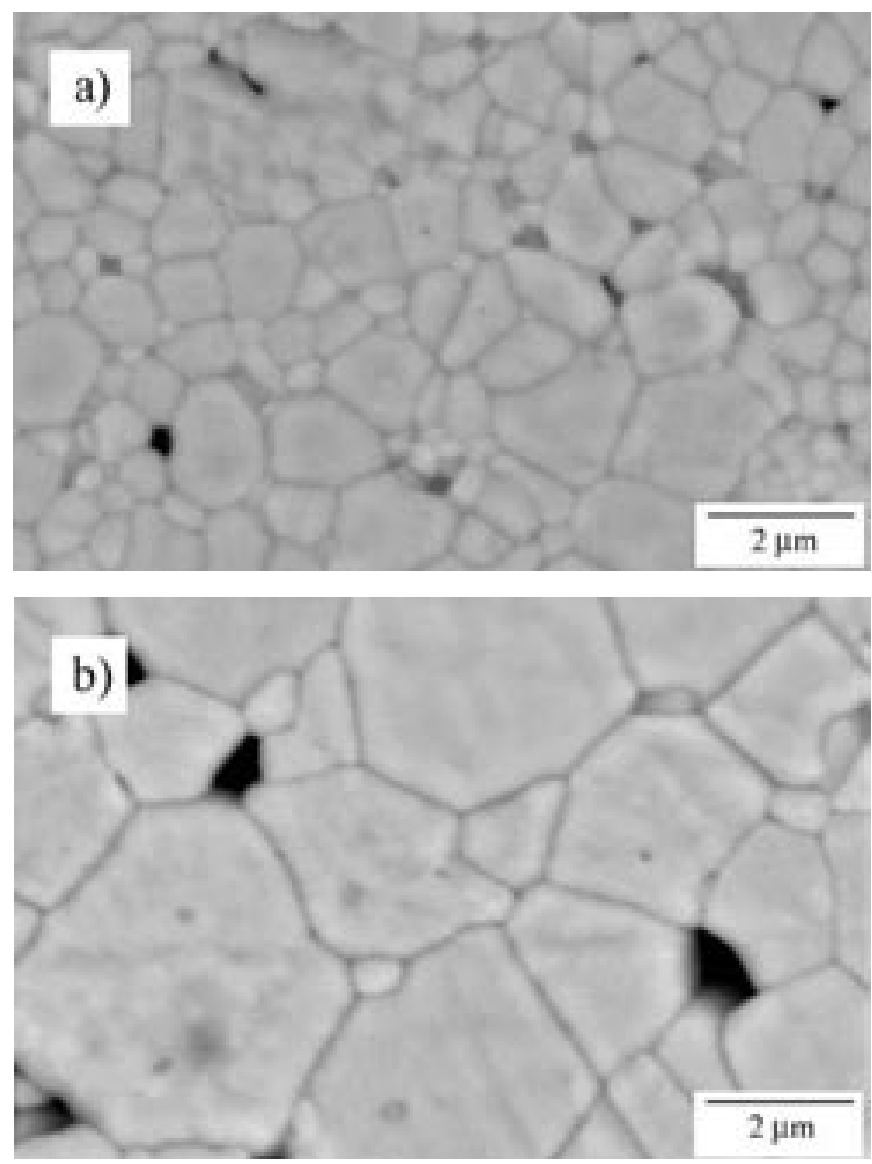

Figura 7. Microestructuras típicas de los polvos de cromita de lantano dopados con Ca y Al, (a) $\mathrm{La}_{0.8} \mathrm{Ca}_{0.2} \mathrm{Cr}_{0.9} \mathrm{Al}_{0.1} \mathrm{O}_{3}$ y (b) $\mathrm{La}_{0.8} \mathrm{Ca}_{0.2} \mathrm{Cr}_{0.95} \mathrm{Al}_{0.05} \mathrm{O}_{3}$ sinterizados en atmósfera de aire, a $1400^{\circ} \mathrm{C}$ durante 5 horas. 


\section{CONCLUSIONES}

En el presente trabajo se logró preparar polvos de cromita de lantano dopados en las posiciones A y B de la estructura $\mathrm{ABO}_{3}$ con adiciones de $\mathrm{Ca}^{2+}$ y $\mathrm{Al}^{3+}$ mediante el método de coprecipitación en medio alcalino. Durante la coprecipitación del gel precursor multicomponente $\left(\mathrm{La}^{3+}, \mathrm{Ca}^{2+}, \mathrm{Cr}^{3+}, \mathrm{Al}^{3+}\right)$ se observó que a un valor de $\mathrm{pH}$ de 13.1, los hidróxidos de cromo y aluminio se disuelven en la solución alcalina de $\mathrm{NaOH}$. Esto permitió que al agregar la solución conteniendo los iones $\mathrm{La}^{3+}$ y $\mathrm{Ca}^{2+}$ precipitara el gel complejo con los cuatro elementos en la estructura. El tratamiento térmico de cristalización a $900^{\circ} \mathrm{C}$ por 3 horas favoreció la formación de polvos con tamaños de partícula inferiores a $0.45 \mu \mathrm{m}$ para todas las soluciones sólidas. Los polvos del tipo $\mathrm{La}_{0.8} \mathrm{Ca}_{0.2} \mathrm{Cr}_{1-\mathrm{x}} \mathrm{Al}_{\mathrm{x}} \mathrm{O}_{3}$ presentaron una buena sinterabilidad a una temperatura de $1400^{\circ} \mathrm{C}$ en atmósferas oxidantes. Los cuerpos sinterizados alcanzaron una densidad real mayor al $95 \%$ de la densidad teórica del compuesto $\mathrm{LaCrO}_{3}$.

\section{AGRADECIMIENTOS:}

Los autores agradecen el apoyo recibido con el proyecto CONACyT clave 38430-U, J.C.R.A. y J.L.R.G. agradecen el apoyo recibido a través del SNI y CINVESTAV-IPN. Otro de los autores, L.M.V.F. agradece el apoyo del CONACyT por la beca otorgada para realizar los estudios de maestría. Los autores agradecen al Ing. Felipe Márquez Torres por su amplia colaboración en la preparación y observación de las muestras en el MEB.

\section{BIBLIOGRAFÍA}

1. O. Yamamoto. "Solid oxide fuel cells: Fundamentals aspects and prospects". Electrochemical Acta. 45 (2000). 2423-2435.

2. S.C. Singhal. "Advances in solid oxide fuel cell technology". Solid state ionics. 13 (2000). 305-313.

3. H.C. Graham and H.H. Davis. "Oxidation/vaporization kinetics of $\mathrm{Cr}_{2} \mathrm{O}_{3}$ ". J. Am. Ceram. Soc. 54 [2] (1971) 89-93.

4. B.F. Flandermeyer, M.M. Nasrallah, D.M. Sparlin, and H.U. Anderson. “High temperature stability of magnesium-doped lanthanum chromite". High Temperature Science, 20 (1985). 259-269.

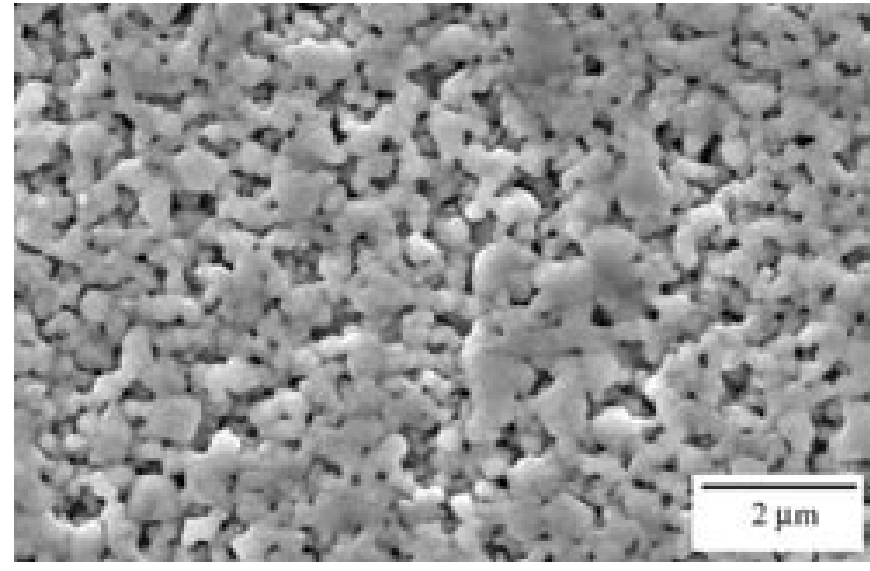

Figura 8. Microestructura, de polvos de cromita de lantano dopados con $10 \%$ molar de $\mathrm{Al}$, sinterizados a $1400^{\circ} \mathrm{C}$ por 5 horas en atmósfera de aire.

5. P.J. Gellings, H.J.M. Bounwmeester. "The CRC handbook of solid state electrochemistry". Ed. CRC Press, Inc. (1997). 422-424.

6. P.A. Lessing. "Mixed-cation oxide powders via polymeric precursors". Bull. Am. Ceram. Soc. 68 (1989), 1002-1007.

7. S. Bilger, G. Blab, R. Forthmann. "Sol-Gel synthesis of lanthanum chromite powder". J. Epn. Ceram. Soc. [17] (1997). 1027-1031.

8. M. Mori, N. Sammes. "Sintering and thermal expansion characterization of Al-doped and Co-doped lanthanum strontium chromites synthesized by the Pechini method". Solid state ionics. 146 (2002). 301-312.

9. M.R. De Guire. "Coprecipitation synthesis of doped lanthanum chromite". J. Mater. Res. [8]. (1993). 2327-2334.

10. M. Inagaki, O. Yamamoto, M. Hirohara. "Complex precipitation synthesis of $\mathrm{LaCrO}_{3}$ and its electrical conductivity". J. Jpn. Ceram. Soc. 98[7]. (1990). 675-678.

11. N. Sakai, T. kawada, H. Yokokawa, M. Dokiya, I. Kojima. “Liquid-phase-assisted sintering calcium-doped lanthanum chromites". J. Am. Ceram. Soc. 76 [3] (1993) 609-616.

12. L. Chick, J. Liu, J. Stevenson, T. Armstrong, D. Mc Cready, G. Maupin, G. Coffey, C. Coyle, "Phase transitions and transient liquid-phase sintering in calcium-substituted chromite", J. Am. Ceram. Soc, 80[8] (1997) 2109-2120.

13. G.M. Christie, P.H. Middleton, B.C.H. Steele. "Liquid phase sintering, electrical, conductivity, and chemical stability of lanthanum chromite doped with calcium and nickel". J. Epn. Ceram. Soc. (1994).14163-14175.

14. M. Mori, Y. Hiei, T. Yamamoto. "Control of thermal expansion of strontiumdoped lanthanum chromite perovskites by B-site doping for high-temperature solid oxide fuel cell separators". J. Am. Ceram. Soc. 84 [4] (2001). 781-786.

15. T. Kikkawa, M. Yoshinaka, K. Hirota, O. Yamaguchi. "Synthesis of $\mathrm{LaCrO}_{3}$ by the hydrazine method". J. Mat. Sci. Lett. 14 (1995). 1071-1073.

Recibido: 22.10 .03

Aceptado: 22.03 .04 


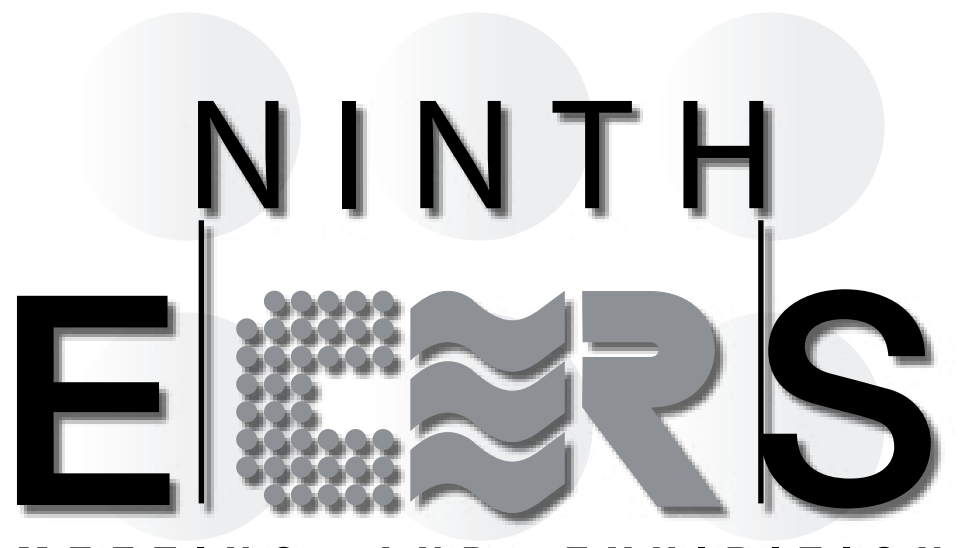

MEETING AND EXHIBITION

\section{CONFERENCE \& EXHIBITION OF THE EUROPEAN CERAMIC SOCIETY}

19-23 JUNE, 2005

PORTOROŽ, SLOVENIA

\section{Invitation}

European R\&D on advanced and traditional ceramics and glasses is becoming increasingly dynamic and innovative. Thousand of fascinating ideas, results and materials are coming from academic and industrial laboratories across the continent. At the same time, European laboratories are enthusiastically forging links with each other and with partners around the world.

Interaction is becoming a key word in European science. The conference of the European Ceramic Society are a traditional forum for researchers, not only from Europe, but also from other parts of the world. The conference is focused on attracting contributions on all aspects of ceramic and glass investigations as well as inorganic nanomaterials and characterisation methods. Its aim is to provide timely, authoritative discussions on new achievements, developments and on-going research in already established, but also emerging, fields.

Leading-edge invited contributions will provide a concise overview of rapidly developing areas of the discipline, while high-quality refereeing of the submitted contributions will ensure the high standards of the proceedings.

Looking forward to seeing you in Portoroû,

\section{Danilo Suvorov}

Chair of the Conference

Profesional Congress Organizer

\section{Chairman:}

Danilo Suvorov

Jožef Stefan Institute, Jamova 39,

SI-1000 Ljubljana, Slovenia

Tel: +38614773871

danilo.suvorov@ijs.si

\section{Conference Office:}

Maja Šimaga

Jožef Stefan Institute, Jamova 39, SI-1000 Ljubljana, Slovenia

Tel: +38614773481

Fax: +38614773875

ecers@ijs.si | www.ecers2005portoroz.com 